\title{
Dietary Calorie Restriction as an Epigenetic Rejuvenator in Aging- Induced Decline of Cognitive Function
}

\section{Mrinal K Poddar* and Apala Chakraborty}

Department of Pharmaceutical Technology, Jadavpur University, Kolkata, India

*Corresponding Author: Prof. (Dr.) Mrinal K Poddar, Department of Pharmaceutical Technology, Jadavpur University, Kolkata, India.

Received: August 28, 2019; Published: September 20, 2019

\begin{abstract}
In recent past, epigenetic regulaions on mammalian aging has set a footprint in geriatric research at the molecular and cellular level. Epigenetic changes are phenotypic changes that are heritable through generations without any alteration of genetic sequences. Epigenetic involvement in memory formations and memory consolidation have been established in recent past. Also, it has been found that stress (due to aging or diseased condition)-induced oxidative damage can cause epigenetic modulation which aggravates memory impairment by initiating changes at the levels of genetic and biochemical profile(s). Epigenetic modulation at the levels of DNA methylation, histone acetylation and micro RNAs modifications have been found to be linked with cognitive impairment during normal as well as diseased aging. Calorie restriction in diet can attenuate aging-induced memory impairment by directly working on the epigenetic biomarkers related to DNA methylation, Histone acetylation and microRNAs level. However, effect of CR diet over disease-induced memory impairment is not well investigated and needs further research. In this review article, we have summarized various epigenetic biomarkers related to cognitive performance during aging and effect of CR diet as an epigenetic modulator/rejuvenator over aging-induced cognitive decline.
\end{abstract}

Keywords: Aging; Calorie Restriction; Cognitive Function; DNA Methylation; Epigenetic

\section{Abbreviation}

ACC: Anterior cingulate cortex; AD: Alzheimer's disease; APP: Amyloid precursor protein; Arc DNA: Activity regulated cytoskeleton DNA; ApoE4: Apolipoprotein epsilon 4; BACE1: Beta-secretase 1; Bcl-2: B-cell lymphoma 2; BCC1: Basal cell carcinoma susceptibility to 1; BDNF: Brain derived neurotropic factor CA1: Cornu Ammonis 1(Hippocampal subfield); CA2: Cornu Ammonis 2(Hippocampal subfield); CA3: Cornu Ammonis 3(Hippocampal subfield); cAMP: Cyclic adenosine monophosphate; CaN: Calcineurin; CBP: CREB binding protein; CG: Cytosine guanine nucleotide, $\mathrm{CH}$ : Non(CG) nucleotide; COMT: Catechol-O-methyltransferase; CR: Calorie restriction; CREB: cAMP response element-binding protein; DG: Dentate gyrus; DNA: Deoxyribonucleic acid; DNAm: DNA methylation; Dnmt: DNA methyltransferase; Dnmt1: DNA methyltransferase 1; Dnmt3a: DNA methyl transferase 3 alpha; Dnmt3b: DNA methyltransferase 3 beta; DS: Down syndrome; DSCAML1: Down syndrome cell adhesion molecule like 1; Fbln2: Fibulin 2; Gabra5: Gamma-aminobutyric acid type A receptor alpha 5 subunit; 5-hmC: 5-hydroxymethylcytosine; H3K4me3: Trimethylation to the lysine 4 on the histone H3 protein; H4K12: Lysine 12 on histone H4; H3K14: Lysine 14 on histone H3; HAT: Histone acetyltransferase; HD: Huntington's disease; HDAC: Histone deacetylase; HDAC2: Histone deacetylase 2; H3S10: H3 serine 10; Hspa5: Heat shock protein family A (Hsp70); KIAA1644: Protein shisa-like-1; 5-mC: 5-Methylcytosine; MF: Mossy fiber; miR-181a-1: microRNA 181a-1; miR-192-5 p: microRNA 192-5 p; miR-30e: MicroRNA-30e; miR-34a: MicroRNA-34a; miR-98-3p: MicroRNA-98-3p; mRNA: Messenger RNA; mthtt: Mutant huntingtin; NP: Neuropsin; Npas4: Neuronal PAS domain protein 4; p300: p300 HAT; PD: Parkinson's disease; PIK2: Polo like kinase 2; PP1: Protein phosphatase I; PS1: Presenilin 1; PS2: Presenilin 2; RNA: Ribonucleic acid; RND1: Rho family GTPase 1; RNS: Reactive nitrogen species; ROS: Reactive oxygen species; SAM: S-adenosyl-L-methionine; Syn: Synapsin; TET1: Tet methylcytosine dioxygenase 1; TET3: Tet methylcytosine dioxygenase 3 ; TGF- $\beta 1$ : Transforming growth factor beta 1 ; TSC2: Tuberous sclerosis complex 2 .

\section{Introduction}

Aging is a progressive decline of biochemical and physiological functions which ultimately results in to death [1]. Several scientists have postulated that aging is a progressive accumulation of damage which is genetically programmed or a result of decline in maintenance [2,3]. Aging at cellular and molecular level is also considered as an individual or combinatorial effect of any of these following nine hallmarks such as epigenetic alterations, mitochondrial dysfunction, cellular senescence, telomere attritions, deregulated nutrient sensing, genomic instability, loss of proteolysis, stem cell ex- 
haustion, and altered intercellular communication as described by Lopez-Otin., et al. [4]. Mammalian aging is inevitably accompanied by structural and neurophysiological changes in the brain which likely to lead towards different neuronal disorders, cognitive decline and other behavioral changes etc. [5,6]. One of the major and fundamental risk factor for acceleration of aging and its related neuronal disorders is stress $[7,8]$. Stress-induced excess accumulation of reactive oxygen and nitrogen species (ROS and RNS respectively) play a role in degeneration of neuronal lipid, protein, and nucleic acids leading to neurodegenerative disorders and/or brain aging [9]. In this context it is important to mention that venerability of neurons towards oxidative damage has been found to be selective and region specific, probably causing selective functional decline of the brain during normal aging or different neuronal disorders [9]. It has been found that entorhinal cortex, hippocampus CA1 region, frontal cortex, and amygdala associated with memory and Alzheimer's disease (AD) like neuronal disorders during aging are maximum susceptible to oxidative damage [10-12]. May be why memory impairment is well observed in aging and its associated disorders. Genetic variations such as (a) decrease in level of cAMP response element binding (CREB) protein which is responsible for long lasting changes in synaptic plasticity and synaptic efficacy related to memory and learning [13] (b) genetic mutation of amyloid precursor protein (APP), presenilin 1 (PS 1) and presenilin 2 (PS 2) [14,15] (c) ablation of neurosis (NP) gene which is predominant in hippocampal CA sub regions, amygdala and also in prefrontal cortex [16-18] have been found to be responsible for aging-induced memory impairment and AD. The genetic changes are often controlled by the "master switches" i.e. epigenomic modulations [19]. In last few years epigenetic regulations have been found to play a crucial role in control of neurotransmitter expressions, neuroinflammation and neurodegeneration [20,21]. "Epigenetic" is comparatively a new and wider approach than genetics as described by Waddington C.H., as "epigenetics" usually covers phenotypic changes which are heritable through generations without any alteration of genetic sequences [22]. It has been also found that stress-induced epigenetic changes associated with neuronal mechanisms has the ability to become trans-generational but therapeutic modulation to protect epigenetic pathways from environmental stimuli is also possible [23,24]. These changes are mainly involved the Chromatin, DNA and RNA modifications which play a crucial role in translations and transcription of signals during normal aging as well as diseased aging [25]. For example, among several epigenetic variations in the brain, bidirectional change in DNA methylation (DNAm) is one of the prominent phenomena which has been observed during normal aging process globally $[26,27]$. Further, this DNA-methylation at cortical region of the brain has been found to play a pivotal role in long-term memory maintenance [28]. Substantial loss of memory is an early sign of neurodegenerative disorders like AD in elderly individuals [29]. Scarpa., et al. [30] and Fuso., et al. [31] have shown that amelioration of impaired memory can be achieved by increasing methyl donor, S-adenosyl-L-methionine (SAM) which can reduce the expression of AD risk gene, APP and PS1, also reduces the aging-related memory impairment. This correlation between genetic and epigenetic variation related to aging associated memory impairment which in advance stage leads to AD like neuronal disorders. Alteration at the epigenetic level could possibly control the genetic expression responsible for such diseased condition at an early stage. As per the report of Perugino., et al. [32] demented population will be 115 million by 2050 globally, demanding an effective therapeutic approach to fight against this global epidemic crisis. A major part of scientific community believes that the answer of this problem and its solution both are related to "lifestyle" including food habits [33]. It has been proved that simple life style changes like calorie restriction (CR) in diet has beneficial effect on memory impairment and other neurodegenerative disorders [34]. Recently, it has been found that CR diet mediated improvement in memory function could be a possible outcome of epigenetic modulation [35]. Here in this article, all the possible epigenetic reasons of memory impairments during normal aging in comparison to disease-induced neurodegenerative conditions are discussed with a special emphasis on dietary calorie restriction as an epigenetic modulator/revitalizer for memory function.

\section{Epigenetic biomarkers responsible for memory formation}

Gene expression mediated structural and functional changes of the neuronal networks are essential for memory formation as well as its functions [36,37]. Epigenetic modulators act as key controller of gene related pathways [38-40]. To study aging-related genetic alteration via epigenetic targeting can be of interesting approach towards recovery from aging-induced degenerations. The complex molecular mechanism of memory formation has partially identified so far until recently when Weng., et al. [41] identified that neuronal activity-dependent transcription factor (Npas4) is required as regulator of MF - CA3 connection (the connection between mossy fiber (MF) inputs from dentate gyrus granule cells and the hippocampal CA3 region) for controlling polo-like kinase (PIK2) expression. This synaptic regulation is important factor for formation of experiencedependent contextual memory formation [42]. This hippocampus mediated task (Morris water maze, contextual fear conditioning novel object recognition etc.) associated with memory formation has been found to be linked with euchromatin related post-translational modifications of histones which is increasing globally [43]. Levenson., et al. [44] and others [45,46] have observed that hippocampus memory task increases the levels of (a) both acetylation at H3 lysine 14 (H3K14) and H4 lysine 12 (H4K12) [44], (b) phosphorylation at $\mathrm{H} 3$ serine 10 (H3S10) [45], and (c) trimethylation at $\mathrm{H} 3$ lysine 4 (H3K4me3) [46] in the hippocampus. In addition it may be mentioned that Miller and Sweatt (2007) first time have observed an increase in DNA methylation associated enzyme level at memory-suppressor gene protein phosphatase I (PP1) along with a decrease of the same at plasticity-associated gene, reelin during learning and memory formation [47]. Later, Lubin., et al. [48] and Munoz., et al. [49] have reported that DNAm at BDNF, Arc, and calcineurin (CaN) genes is also associated with memory formation and its maintenance. These give evidences of epigenetic control over synaptic plasticity and memory related neuronal gene expressions 
[48,49]. Sharma., et al. [50] have shown that membrane depolarization related to memory formation is down regulated by Dnmt1 and Dnmt3a in the cortical region and hence further justify the role of DNAm in memory formation.

Memory persistence is another important aspect of memory formation as well as its function which is a resultant effect of altered synaptic strength of complex neuronal circuit although it needs further clarifications [51]. It has been speculated for a long time back that DNA might be the store house of memory [42]. The function of DNA methylation and participation of anterior cingulate cortex (ACC) in memory maintenance have been identified by Miller, et al. [28] on 2010. They have also observed that administration of Dnmt inhibitor (5-azadeoxycytidine or zebularine) into the ACC after 29 days of memory training, DNA methylation is reduced on CaN gene with a memory impairment suggesting a role of DNA methylation and/or demethylation balance in ACC region mediated memory maintenance and storage [28].

\section{Aging, memory impairment and epigenetic modulation}

Aging causes deterioration in synaptic functions of the hippocampus and pre frontal cortex, the regions which are often correlated with cognitive impairment. Association of reduction in DNAm with aging is an well observed fact [52]. Recent observation in human model reveal that chronological alteration in gene expression can be correlated with alteration of DNAm with age as well as aging at the regulatory regions of these genes [53]. There are several evidences of links between DNAm and cognitive impairment during aging in different mammalian groups such as in aged rats the decrease in methylation in (a) Arc DNA at hippocampal CA1 and Dentate gyrus (DG) regions [54], (b) promoter regions of Gabra5, Hspa5, and Syn etc. [55] and (c) increases in the levels of 5-methylcytosine ( 5 - $\mathrm{mC}), 5$-hydroxymethylcytosine (5 - hmC) has been observed [27]. In mice model, loss of DNA methyltransferases (Dnmt1, Dnmt3a, Dnmt3a2) in the adult brain cause cognitive deficit [56]. In this context, it may be stated that memory impairment has been found in both mutant Tet1 animals [57] and in Dnmt1 mutated humans also [58]. Apart from DNAm, chromatin remodeling via histone acetylation by histone deacetylase (HDAC) inhibition has been also found to regulate synaptic and cognitive function during aging [59]. Aging associated Histone acetylation at lysine 12 on histone H4 (H4K12) in hippocampus has been found to decrease in learning-induced hippocampal gene transcription [60]. Similarly, the up-regulation of transcriptional marker H3K$4 \mathrm{me} 3$ has been found to be linked with memory impairment in aging [61]. The above evidences [58-61] suggest that there is a participation of histone acetylation along with DNAm in aging-induced cognitive impairments. Another probable epigenetic biomarker of aging-induced cognitive impairment is microRNA (miRNA) [6264]. Some miRNAs have been found to be correlated with cognitive impairment related to depression and aging. Such as, up-regulation of miR192 - 5 p has been found to attenuate cognitive impairment in depressed mice via fibulin 2 (Fbln2)-mediated transforming growth factor beta 1 (TGF- $\beta 1$ ) signaling pathway [62] whereas down-regulation of $\mathrm{miR}-34 \mathrm{a}, \mathrm{miR}-30 \mathrm{e}$ and $\mathrm{miR}$ - 181a - 1 increase the B-cell lymphoma 2 (BCL2) mediated cell apoptosis followed by neuronal cell death [63]. Although the involvement of miRNAs in aging related cognitive impairment is not fully understood [Figure $1(\mathrm{a})]$ and need further investigations.

Role of epigenetic alteration on memory impairments during aging related neuronal disorders

As per the study of Rani., et al. [64] Montreal Cognitive Assessment (MoCA) has revealed that 13 miRNAs are negatively correlated with aging related cognitive decline although the same miRNAs are not linked with AD like neurodegenerative diseases. Memory impairment during neurodegenerative disorders (such as AD and Huntington's disease (HD) etc.) is also an expected outcome of epigenetic deregulations. It has been observed that $\mathrm{AD}$ related neurons contain significantly larger amount of cluster of hypomethylated enhancers in the Down syndrome cell adhesion molecule like 1 (DSCAML1) gene that targets Beta-secretase 1 (BACE1), which by up regulation increases amyloid plaques deposition, neurofibrillary tangles, and cognitive impairment [65]. It has been also observed that later stage of AD related increase in Histone deacetylase 2 (HDAC2) level reduces histone acetylation which could be correlated with impairment of synaptic plasticity and learning and memory [66-68]. So it can be speculated that HDAC2 knockdown can work as a tool for neurodegeneration associated memory impairment by increasing RNA polymerase and gene expression of memory and learning functions [68]

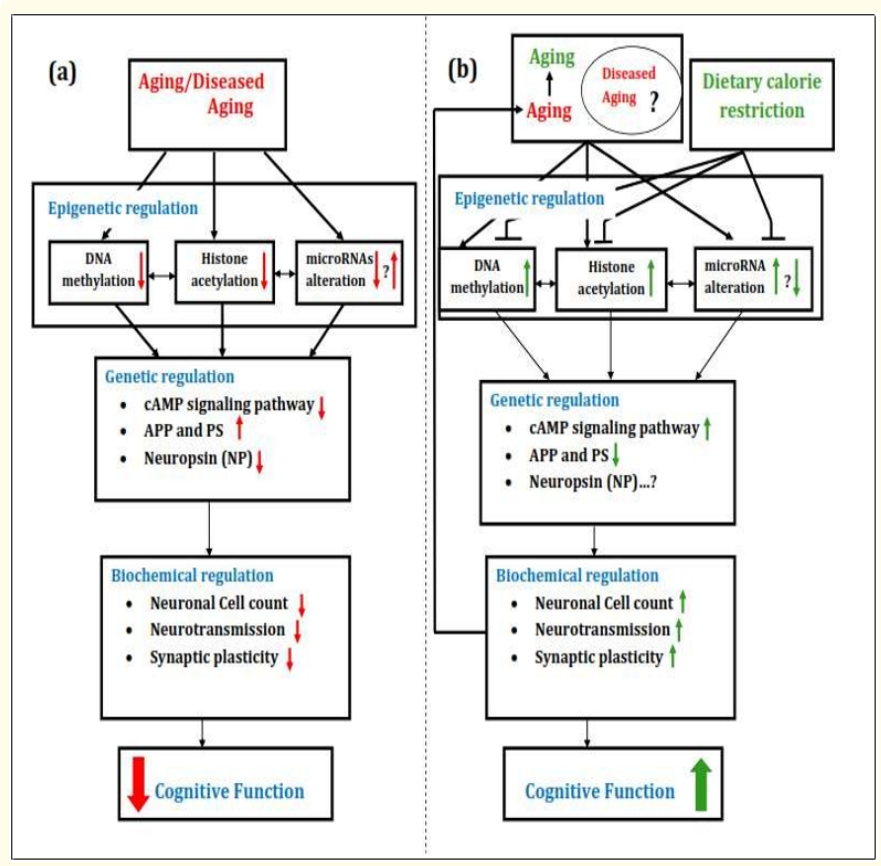

Figure 1: Possible mechanism of epigenetic action of (a) aging/ diseased aging on declination of cognitive function (b) dietary calorie restriction on aging-induced impairment of cognitive function.

APP: Amyloid precursor protein; cAMP: Cyclic adenosine monophosphate; NP: Neuropsin; PS: Presenilin. Red Arrow( $(\downarrow)$ indicates down-regulation and Green Arrow ( $\uparrow$ ) indicates up-regulation. 
In HD patients, the sequestration of CREB binding protein (CBP) by mutant huntingtin (mthtt) has been found to promote neuronal transcriptional dysfunction by blocking its (CBP) co-factor activity during histone acetylation [69]. It is known that CBP works as a transcriptional cofactor of histone acetyltransferase (HAT) during chromatin remodeling [70] and loss of CBP function is indirectly responsible for alteration in acetylation level of histones and chromatin structure in neuron [69, 71]. Korzus., et al. [72] have found that impairment of short term to long term memory conversion is related to the reduction in HAT activity. The disability in performing long term memory recognition tests by p300 mutant mice (CBP homologue) having lack of active HAT [73] suggests that there may be an epigenetic link between cognitive dysfunction during HD and reduced CBP-HAT activity [74].

Down syndrome (DS) related cognitive impairment has been found to be linked with DNA methylation. In DS, comparing the aging-induced memory impairment and corresponding age-matched control group, Jones., et al. have shown that DNA methylation occurs in 5 probes (BICC1, KIAA1644, RND1, TSC2 (two isoforms)) related to cognitive impairment with respect to their corresponding control groups. Further, it may be also mention that among those 5 probes TSC2 gene is a component of mTOR pathway, linked with Alzheimer's [75].

One of the predominant non-motor dysfunction related to Parkinson's disease (PD) is cognitive impairment. Cognitive impairment in PD is heterogeneous and varies from subtle to mild and up to dementia [76]. Cognitive deficits during PD typically affect attention, visuospatial function, and speed of processing [77]. The mechanism of cognitive deficit during PD is not fully understood although presence of dual hypothesis has been suggested one of which, is (a) a frontostriatal/executive function and its related dopamine depletion which interacts with the catechol-0-methyltransferase (COMT) genotype but its progress towards dementia is optional [78] and the other one is (b) involvement of AD pathology, non-dopaminergic transmitters, and apolipoprotein epsilon 4 (ApoE4) genotype due to dysfunction of posterior cortical followed by impairment of language/semantic fluency, and visuospatial orientation/pentagon copying [79]. However, the epigenetic involvement on cognitive impairment during PD has not been evaluated so far and need further investigations. These undefined signaling pathways have not been shown in the flow sheet [Figure 1(a)] of the present article. Future studies with other variations of neurodegenerative diseases linked to aging processes related the changes in epigenetic factors will explore our knowledge in this specialized field of geriatric research.

Calorie Restriction as a revitalizer of memory at the epigenetic level during aging

Dietary calorie restriction (CR) a reduced dietary energy intake (usually 20-40\%), without compromising the micronutrient content, has been proved to be beneficial on aging-related health disorders [80]. An important property of CR is to generate a cellular memory which stays even after the discontinuation of calorie restricted diet [81-83]. Hahn., et al. [84] and others [25, 85] have investigated that changes in DNA methylation induced by CR persist at least $20-50 \%$ for 2 months in rodents even after discontinuation of CR after 1 month of its consecutive supplementation. Surprisingly, this phenomena has found to be effective in humans as these epigenetic down regulation in DNAm persist for six decades after prenatal exposure to hunger during Dutch Hunger Winter in $1944-1945$ [86]. This particular incident further justifies CR as a probable epigenetic modulator. In recent past, the epigenetic modulatory property of CR during aging has been investigated and opened up a new insight in this field at the cellular and molecular levels [87-89]. Heijmans., et al. [86] and other investigators [87,90] have reported that CR has the property to reverse DNA methylation patterns during aging along with that helps to maintain chromatin function and also increase genomic stability. Hadad., et al. [35] have observed that hippocampal DNA methylation pattern using CR fed "genome-wide bisulfite sequencing" in CR fed young (3 months) and aged (24 months) mice is altered at CG and CH sites irrespective of age. Their study also provides information that $\mathrm{CR}$ regulates aging-induced methylation by CR specific routes such as DNMT1 and TET3 promoters for hypermethylation than those affected by aging only or shows neuroprotective effect by both the signaling pathways [35]. In this context, it may be mentioned that Meyerson., et al. [91] as well as others [92,93] have shown that HDACs activities also increase during CR supplementation and helps to promote longevity. Recently transcriptome analysis has further shown that CR diet consumption overexpresses neuroprotective gene in rat cerebral cortex possibly by increasing miRNA ( $\mathrm{miR}-98-3 p)$ which maintains the homeostasis of HDAC and histone acetyltransferase [89]. In mice brain Khanna., et al. [63] have shown that the counter acting effect of CR diet on aging induced increase in miR-181a-1, miR-30e, and miR - 34a leads to elevate Bcl- 2, which is involve in apoptosis of healthy neuronal cell and hence indirectly suggests a possible neuroprotection [94]. So, it is not unlikely to hypothesize that CR diet can protect memory impairment by maintaining HDAC and histone acetyltransferase homeostasis in the cerebral cortex, as cortical region has an active role in long-term memory consolidation. Recent past evidences of Chouliaras., [27,95] et al have shown that CR diet attenuates aging-induced impairment of cognitive function [6] by increasing 5 - $\mathrm{mC}$ and Dnmt3A in hippocampal CA3 and CA1 - 2 regions respectively [Figure 1(b)]. As discussed earlier DNA methylation has a role to play in formation of memory, it can be stated that these two phenomena are correlative. Though the exact mechanism by which CR diet attenuates the hippocampal 5 - $\mathrm{mC}$ and Dnmt3A in aged brain is not fully known it may be suggested that CR diet is definitely involved with (a) one carbon metabolic pathway and control the methyl-groups availability [96] (b) homeostasis between methylation and hydroxy methylation $[84,95,96]$ (c) histone deacetylases (by sirtuins pathway) and increase life span [97] and (d) chromatin remodeling [98] [Figure 1(b)].

All the above mentioned observations clearly state that dietary CR has a positive effect on cognitive function by epigenetic up-regulation in normal aging; however there is a gap of information re- 
garding effect of calorie restriction on memory impairment during diseased aging. Though CR has been found to attenuate the effect of neurodegenerative disorders at the epigenetic level in AD, PD, HD etc. during aging $[80,99,100,101]$ [Figure 1 (a)] there is a lack of information in relation to epigenetic regulation by CR diet that can directly attenuate the cognitive impairment during diseased aging which needs a proper investigations with a scientific evaluation.

\section{Conclusion}

Bridging between epigenetic and transcriptional changes is crucial for better understanding of neuronal aging process and also for achieving a better therapeutic regimen for healthy living [102]. The beauty of energy restriction in diets is the effectiveness of CR over board spectrum physiological abnormalities during aging including cognitive function [102]. Dietary CR is non-invasive, costeffective and easily accessible and can be accepted by global population. Involvement of calorie restriction on alteration of epigenetic factor(s) not only validates the acceptability of such regimen as an anti-aging therapy, but also opens-up newer challenge(s) towards more advance and precise application of CR diet to achieve its maximum effect. Needless to mention that there is still some space to be evaluated and established on CR diet's affectivity in epigenetic signaling pathways related to cognitive impairment during aging and especially in diseased aging. Effectiveness of CR on diseaserelated epigenetic alteration in memory impairment during aging is lacking in the frontiers of nutritional neuroscience and need further investigations. Moreover, the optimization of calorie intake with respect to dose and duration for converting it (CR diet) in a therapeutic regimen is also essential which in fact in future this approach will help to transfer from bench to bedside with maximum beneficial effect as rejuvenator for memory preservation as well as its function during aging.

\section{Acknowledgements}

Authors are thankful to Dr. Soumyabrata Banerjee, NPDF, Department of Pharmaceutical Technology, Jadavpur University for his help during preparation of Figure 1 of this article. Authors are also thankful to University Grant Commissions, New Delhi, India; Department of Pharmaceutical Technology, Jadavpur University, Kolkata, India and Indian Council of Medical Research, New Delhi, India for providing facilities and support.

\section{Disclosers}

No conflicts of interest are declared by the authors.

\section{Bibliography}

1. Goldsmith TC. "On the programmed/non-programmed aging controversy". Biochemistry (Moscow) 77.7 (2012): 729-732.

2. Blagosklonny MV. "Aging is not programmed". Cell Cycle 12 (2013):3736-3742.

3. Kowald A and Kirkwood TBL. "Can aging be programmed? A critical literature review”. Aging Cell 15 (2016): 986-998.
4. Lopez-Otin C., et al. "The hallmarks of aging". Cell 153.6 (2013): 1194-1217.

5. Glisky LE. "Changes in Cognitive Function in Human Aging". In: Riddle DR, editor. Brain Aging: Models, Methods, and Mechanisms. Boca Raton (FL): CRC Press/Taylor and Francis; Chapter 1 (2007).

6. Das A., et al. "Role of physical activity and calorie induced changes in cognition and depression of young and aged humans". The Indian Journal of Nutrition and Dietetics 54.3 (2017): 316-335.

7. Zannas AS., et al. "Stressful life events, perceived stress, and 12-month course of geriatric depression: direct effects and moderation by the 5-HTTLPR and COMT Val158Met polymorphisms". Stress 15 (2012): 425-434.

8. Peavy GM., et al. "Effects of chronic stress on memory decline in cognitively normal and mildly impaired older adults". American Journal of Psychiatry 166 (2009):1384-1391.

9. Xinkun W and Michaelis EK. "Selective neuronal vulnerability to oxidative stress in the brain". Frontiers in Aging Neuroscience 2.12 (2010).

10. Hyman BT., et al. "Alzheimer's disease: cell-specific pathology isolates the hippocampal formation". Science 225 (1984): 1168-1170.

11. Braak $\mathrm{H}$ and Braak E. "Neuropathological stageing of Alzheimer-related changes". Acta Neuropathol 82 (1991): 239-259.

12. Terry RD., et al. "Physical basis of cognitive alterations in Alzheimer's disease: synapse loss is the major correlate of $\operatorname{cog}$ nitive impairment". Annals of Neurology 30 (1991): 572-580.

13. Paramanik V and Thakur MK. "Role of CREB signaling in aging brain”. Archives Italiennes de Biologie 151 (2013): 33-42.

14. Sivanandam TM and Thakur MK. "Amyloid precursor protein (APP) mRNA level is higher in the old mouse cerebral cortex and is regulated by sex steroids". Journal of Molecular Neuroscience 43 (2011): 235-240.

15. Ghosh S and Thakur MK. "PS1 expression is downregulated by gonadal steroids in adult mouse brain". Neurochemical Research 33 (2008): 365-369.

16. Chen ZL., et al. "Expression and activity-dependent changes of a novel limbic- serine protease gene in the hippocampus". Journal of Neuroscience 75 (1995): 5088-5097.

17. Suzuki J., et al. "Ontogeny of neuropsin mRNA expression in the mouse brain". Neuroscience Research 23 (1995): 345-351. 
18. Yousef GM and Diamandis EP. "The new human tissue kallikrein gene family: structure, function and association to disease". Endocrine Reviews 22 (2001): 184-204.

19. Konar A., et al. "Age-associated Cognitive Decline: Insights into Molecular Switches and Recovery Avenues". Aging and Disease 7.2 (2016): 121-129.

20. Zannas AS., et al. "Lifetime stress accelerates epigenetic aging in an urban, African American cohort: relevance of glucocorticoid signaling". Genome Biology 16.1 (2015): 1-12.

21. Banerjee K., et al"'. Epigenetic control of neurotransmitter expression in olfactory bulb interneurons". International Journal of Developmental Neuroscience 31.6 (2013): 415-423.

22. Waddington $\mathrm{CH}$. "The epigenotype. 1942". International Journal of Epidemiology 41.1 (2012): 10-13.

23. Irene L and Ventura R. "Epigenetic Inheritance: Concepts, Mechanisms and Perspectives". Frontiers in Molecular Neuroscience 11 (2018): 1-22.

24. Vaiserman A and Pasyukova E. "Epigenetic drugs: a novel anti-aging strategy?”. Frontiers in Genetic 3 (2012): 1-3.

25. Kim-Ha J and Kim YJ. "Age-related epigenetic regulation in the brain and its role in neuronal diseases". BMB Reports 49.12 (2016): 671-680.

26. Bollati V., et al. "Decline in genomic DNA methylation through aging in a cohort of elderly subjects". Mechanisms of Ageing and Development 130 (2009): 234-239.

27. Chouliaras L., et al. "Age- related increase in levels of 5-hydroxymethylcytosine in mouse hippocampus is prevented by caloric restriction". Current Alzheimer Research 9.5 (2012): 536-544.

28. Miller AC., et al. "Cortical DNA methylation maintains remote memory". Nature Neuroscience 13.6 (2010): 664-666.

29. Bertram L., et al. "The genetic epidemiology of neurodegenerative disease". Journal of Clinical Investigation 115.6 (2005): 1449-1457.

30. Scarpa S., et al. "Presenilin 1 gene silencing by S-adenosylmethionine: a treatment for Alzheimer disease?". FEBS Letters 541 (2003): 1873-3468.

31. Fuso A., et al. "S- adenosylmethionine/homocysteine cycle alterations modify DNA methylation status with consequent deregulation of PS1 and BACE and beta-amyloid production". Molecular and Cellular Neuroscience 28 (2005) 195-204.

32. Peracino A and Pecorelli S. "The Epidemiology of Cognitive Impairment in the Aging Population: Implications for Hearing Loss". Audiol Neurotol 21.1 (2016): 3-9.
33. Miley-Akerstedt A., et al. "Lifestyle Factors Are Important Contributors to Subjective Memory Complaints among Patients without Objective Memory Impairment or Positive Neurochemical Biomarkers for Alzheimer's Disease". Dementia and Geriatric Cognitive Disorders Extra 8.3 (2018): 439-452.

34. Witte AV., et al. "Caloric restriction improves memory in elderly humans". Proceedings of the National Academy of Sciences of the United States of America 106.49 (2009): 1255-1260.

35. Hadad N., et al. "Caloric restriction mitigates age-associated hippocampal differential CG and non-CG methylation”. Neurobiology of Aging 67 (2018): 53-66.

36. Davis HP and Squire LR. "Protein synthesis and memory: a review”. Psychological Bulletin Journal 96.3 (1984): 518-559.

37. Bailey CH., et al. "Structural Components of Synaptic Plasticity and Memory Consolidation". Cold Spring Harbor Perspectives in Biology 7.7 (2015): a021758.

38. Blakey CA and Litt MD. "Chapter 1 - Epigenetic gene expression-an introduction". Epigenetic Gene Expression and Regulation. Academic Press (2015): 1-19.

39. Palumbo S., et al. "Genes and Aggressive Behavior: Epigenetic Mechanisms Underlying Individual Susceptibility to Aversive Environments". Frontiers in Behavioral Neuroscience 12 (article no. 117) (2018): 1-9.

40. McGowan PO and Roth TL. "Epigenetic pathways through which experiences become linked with biology". Development and Psychopathology 27.2 (2015): 637-48.

41. Weng FJ., et al. "Npas4 Is a Critical Regulator of Learning-Induced Plasticity at Mossy Fiber-CA3 Synapses during Contextual Memory Formation". Neuron 97 (2018): 1137-1152.

42. Gräff J., et al. "Dynamic histone marks in the hippocampus and cortex facilitate memory consolidation". Nature Communications (2012): 1-8.

43. Zovkic IB., et al. "Epigenetic regulation of memory formation and maintenance". Learning and Memory 20 (2013): 61-74.

44. Levenson JM., et al. "Regulation of histone acetylation during memory formation in the hippocampus". The Journal of Biological Chemistry 279 (2004): 40545-40559.

45. Chwang WB., et al. "ERK/MAPK regulates hippocampal histone phosphorylation following contextual fear conditioning". Learning and Memory 13 (2006): 322-328.

46. Bousiges 0., et al. "Spatial memory consolidation is associated with induction of several lysine-acetyltransferase (histone acetyltransferase) expression levels and $\mathrm{H} 2 \mathrm{~B} / \mathrm{H} 4$ acetylationdependent transcriptional events in the rat hippocampus". Neuropsychopharmacology 35 (2010): 2521-2537. 
47. Miller CA and Sweatt, JD. “Covalent modification of DNA regulates memory formation”. Neuron 53 (2007): 857-869.

48. Lubin FD., et al. "Epigenetic regulation of BDNF gene transcription in the consolidation of fear memory". Journal of Neuroscience 28 (2008): 10576-10586.

49. Munoz PC., et al. "Correlations of recognition memory performance with expression and methylation of brain-derived neurotrophic factor in rats". Biological Research 43 (2010): 251-258.

50. Sharma RP., et al. "Depolarization induces down regulation of DNMT1 and DNMT3a in primary cortical cultures". Epigenetics 3 (2008): 74-80.

51. GuptaAgarwal S., et al. "G9a/GLP histone lysine dimethyltransferase complex activity in the hippocampus and the entorhinal cortex is required for gene activation and silencing during memory consolidation". Journal of Neuroscience 32 (2012): 5440-5453.

52. Numata S., et al. "DNA methylation signatures in development and aging of the human prefrontal cortex". American Journal of Human Genetics 90 (2012): 260-272.

53. Peters MJ., et al. "The transcriptional landscape of age in human peripheral blood". Nature Communications 6 (2015): 1-14.

54. Penner MR., et al. "Age-related changes in Arc transcription and DNA methylation within the hippocampus". Neurobiology of Aging 32 (2012): 2198-2210.

55. Haberman RP., et al. "Characterization of CpG island DNA methylation of impairment related genes in a rat model of cognitive aging". Epigenetics 7 (2012): 1008-1019.

56. Smallwood SA and Kelsey G. "Genome-wide analysis of DNA methylation in low cell numbers by reduced representation bisulfite sequencing". Methods in Molecular Biology 925 (2012): 187-197.

57. Kaas GA., et al. "TET1 controls CNS 5-methylcytosine hydroxylation, active DNA demethylation, gene transcription, and memory formation". Neuron 79.6 (2018): 1086- 1093.

58. Klein CJ., et al. "Mutations in DNMT1 cause hereditary sensory neuropathy with dementia and hearing loss". Nature Genetics 43.6 (2011): 595-600.

59. Vecsey CG., et al. "Histone deacetylase inhibitors enhance memory and synaptic plasticity via CREB: CBP-dependent transcriptional activation". Journal of Neuroscience 27 (2007): 6128-6140
60. Spiegel AM., et al. "Epigenetic contributions to cognitive aging: disentangling mindspan and lifespan". Learning and Memory 21 (2014): 569-574.

61. Gupta S., et al. "Histone methylation regulates memory formation”. Journal of Neuroscience 30 (2010): 3589-3599.

62. Tang CZ., et al. "Up-regulated miR-192-5p expression rescues cognitive impairment and restores neural function in mice with depression via the Fbln2-mediated TGF- $\beta 1$ signaling pathway". The FASEB Journal 33.1(2019): 606-618.

63. Khanna A., et al. "Gain of survival signaling by down-regulation of three key miRNAs in brain of calorie-restricted mice". Aging 3(2011): 223-236.

64. Rani A., et al. "miRNA in Circulating Microvesicles as Biomarkers for Age-Related Cognitive Decline". Frontiers in Aging Neuroscience 9 (2017): 1-10.

65. Li P., et al. "Epigenetic dysregulation of enhancers in neurons is associated with Alzheimer's disease pathology and cognitive symptoms". Nature Communications 10 (2019): 1-14.

66. Fischer A., et al. "Recovery of learning and memory is associated with chromatin remodeling”. Nature 447 (2007): 178182.

67. Ricobaraza A., et al. "Phenylbutyrate ameliorates cognitive deficit and reduces tau pathology in an Alzheimer's disease mouse model". Neuropsychopharmacology 34 (2009): 17211732.

68. Gräff J., et al. "An epigenetic blocked of cognitive functions in the neurodegenerating brain". Nature 483 (2012): 222-226.

69. Alarcón JM., et al. "Chromatin acetylation, memory, and LTP are impaired in $\mathrm{CBP}+/-$ mice: a model for the cognitive deficit in Rubinstein-Taybi syndrome and its amelioration". Neuron 42 (2004): 947-959.

70. Bannister AJ and Kouzarides T. "The CBP co-activator is a histone acetyltransferase". Nature 384 (1996): 641-643.

71. Steffan JS., et al. "Histone deacetylase inhibitors arrest polyglutamine-dependent neurodegeneration in Drosophila". $\mathrm{Na}$ ture 413 (2001): 739-743.

72. Korzus E., et al. "CBP histone acetyltransferase activity is a critical component of memory consolidation". Neuron 42 (2004): 961-972.

73. Oliveira AM., et al. "Transgenic mice expressing an inhibitory truncated form of p300 exhibit long-term memory deficits". Learning and Memory 14 (2007): 564-572. 
74. Ryu H., et al. "ESET/SETDB1 gene expression and histone H3(K9) trimethylation in Huntington's disease". Proceedings of the National Academy of Sciences of the United States of America 103 (2006): 19176-19181.

75. Jones MJ., et al. "Distinct DNA methylation patterns of cognitive impairment and trisomy 21 in Down syndrome". BMC Medical Genomics 6 (2013): 1-11.

76. Broeders M., et al. "Cognitive change in newly-diagnosed patients with Parkinson's disease: a 5-year follow-up study". Journal of the International Neuropsychological Society 19 (2013): 695-708.

77. Hoogland J., et al. "Mild cognitive impairment as a risk factor for Parkinson's disease dementia". Movement Disorders (2017).

78. Williams-Gray CH., et al. "The distinct cognitive syndromes of Parkinson's disease: 5year follow-up of the CamPaIGN Cohort". Brain 132 (2009): 2958-2969.

79. Kehagia AA., et al. "Cognitive impairment in Parkinson's disease: the dual syndrome hypothesis". Neurodegenerative Diseases 11 (2013): 79-92.

80. Van Cauwenberghe C., et al. "Caloric restriction: beneficial effects on brain aging and Alzheimer's disease". Mammalian Genome 27.7-8 (2016): 300-319.

81. Cameron KM., et al. "Male mice retain a metabolic memory of improved glucose tolerance induced during adult onset, short-term dietary restriction". Longevity and Healthspan 1.3 (2012): 1-9.

82. Selman $\mathrm{C}$ and Hempenstall S. "Evidence of a metabolic memory to early-life dietary restriction in male C57BL/6 mice". Longevity and Healthspan 1.2 (2012): 1-11.

83. Sun L., et al. "Life-span extension in mice by preweaning food restriction and by methionine restriction in middle age". Journals of Gerontology Series A: Biological Sciences and Medical Sciences 64 (2009): 711-722.

84. Hahn 0., et al. "Dietary restriction protects from age-associated DNA methylation and induces epigenetic reprogramming of lipid metabolism". Genome Biology 18 (article no 56) (2017): 1-18

85. Unnikrishnan A., et al. "Role of DNA methylation in the dietary restriction mediated cellular memory". Geroscience 39 (2017): 331-345.

86. Heijmans BT., et al. "Persistent epigenetic differences associated with prenatal exposure to famine in humans". Proceedings of the National Academy of Sciences of the United States of America 105 (2008): 17046-17049.
87. Li Y., et al. "Epigenetic regulation of caloric restriction in aging”. BMC Medicine 9 (2011): 1-12.

88. Gensous N., et al. "The Impact of Caloric Restriction on the Epigenetic Signatures of Aging". International Journal of Molecular Sciences 20.8 (2019): 1-14.

89. Wood SH., et al. "Transcriptome analysis in calorie-restricted rats implicates epigenetic and post-translational mechanisms neuro protection and aging". Genome Biology 16 (2015): 1-18

90. Munoz-Najar U and Sedivy JM. "Epigenetic control of aging". Antioxidants and Redox Signaling 14 (2011): 241-259.

91. Meyerson M., et al. "hEST2, the putative human telomerase catalytic subunit gene, is up- regulated in tumor cells and during immortalization". Cell 90 (1997): 785-795.

92. Li Y., et al. "Glucose restriction can extend normal cell lifespan and impair precancerous cell growth through epigenetic control of hTERT and p16 expression". The FASEB Journal 24 (2010): 1442-1453

93. Heldt SA., et al. "Hippocampus-specific deletion of BDNF in adult mice impairs spatial memory and extinction of aversive memories". Molecular Psychiatry 12.7 (2007): 656-670.

94. Maalouf M., et al. "The neuroprotective properties of calorie restriction, the ketogenic diet, and ketone bodies". Brain Research Reviews 59 (2009): 293-315.

95. Chouliaras L., et al. "Caloric restriction attenuates age-related changes of DNA methylltransferase 3a in mouse hippocampus". Brain, Behavior, and Immunity 25 (2011): 616- 623.

96. Fuso A., et al. "DNA methylase and demethylase activities are modulated by one-carbon metabolism in Alzheimer's disease models". The Journal of Nutritional Biochemistry 22 (2011): 242-251.

97. Chen D., et al. "Tissue-specific regulation of SIRT1 by calorie restriction". Genes and Development 22 (2008): 1753-1757.

98. Dang W., et al. "Inactivation of yeast Isw2 chromatin remodeling enzyme mimics longevity effect of calorie restriction via induction of genotoxic stress response". Cell metabolism 19.6 (2014): 952-966.

99. Duan W., et al. " Dietary restriction normalizes glucose metabolism and BDNF levels, slows disease progression, and increases survival in huntingtin mutant mice". PNAS 100.5 (2003): 2911-2916

100. Fuso A and Domenichelli C. "Diet, Epigenetics, and Alzheimer's Disease". Handbook of Nutrition, Diet, and Epigenetics. Springer (2019): 987-1003. 
101. Van Heesbeen HJ and Marten PS. "Entanglement of Genetics and Epigenetics in Parkinson's Disease". Frontiers in Neuroscience 13 (article no 277) (2019): 1-15.

102. Balasubramanian P., et al. "Aging and Caloric Restriction Research: A Biological Perspective with Translational Potential". EBioMedicine 21 (2017): 37-44.

\section{Volume 3 Issue 10 October 2019}

(C) All rights are reserved by Mrinal K Poddar and Apala

\section{Chakraborty.}

